\title{
RFID Application of Smart Grid for Asset Management
}

\author{
Xiwei Wang, ${ }^{1}$ Qi Dang, ${ }^{1}$ Jinglin Guo, ${ }^{1}$ and Hongbin Ge ${ }^{2}$ \\ ${ }^{1}$ Beijing China Power Information Technology Co., Ltd., State Grid Electric Power Research Institute, Haidian District, \\ Beijing 100192, China \\ ${ }^{2}$ Beijing University of Posts and Telecommunications, Haidian District, Beijing 100876, China
}

Correspondence should be addressed to Qi Dang; dangqi@sgepri.sgcc.com.cn

Received 26 August 2013; Accepted 8 October 2013

Academic Editor: Yao Yuan

Copyright (C) 2013 Xiwei Wang et al. This is an open access article distributed under the Creative Commons Attribution License, which permits unrestricted use, distribution, and reproduction in any medium, provided the original work is properly cited.

RFID technology research has resolved practical application issues of the power industry such as assets management, working environment control, and vehicle networking. Also it provides technical reserves for the convergence of ERP and CPS. With the development of RFID and location-based services technology, RFID is converging with a variety of sensing, communication, and information technologies. Indoor positioning applications are under rapid development. Micromanagement environment of the assets is a useful practice for the RFID and positioning. In this paper, the model for RFID applications has been analyzed in the microenvironment management of the data center and electric vehicle batteries, and the optimization scheme of enterprise asset management is also proposed.

\section{Introduction}

With the innovation of technology and applications, RFID requires a combination with other technologies and concepts of innovative design to meet the application requirements in different aspects of the management and production. In this paper, the applications and design for the intensive assets management are proposed, which are from the concrete practice of the enterprise asset management of data center equipment and electric vehicle battery: State Grid Corporation data center construction and electric vehicles network construction \& operation.

1.1. Overview of Application for Life Cycle Asset Management. IT Asset Management based on RFID is a priority for the asset life-cycle management framework. State Grid Corporation of China has launched the construction of the centralized disaster recovery center, the important aspect of which is condition monitoring for environments and equipment in large data center. In the construction of electric vehicle charging service network, battery asset management is an important foundation for electricity service implementation.

The ultra-large-scale data centers require real-time sensing device status in the room and cabinet, focusing on internal regional environmental management, including internal dynamic management of the device in the room, internal static management of the device in the cabinet, and collaborative management of the staff. The battery device management is divided into the storage environment and external operating environment. Each battery data of the battery management system in a warehouse environment can be collected in real time. Real-time status of the battery in car, obtained by means of the vehicle terminal in the running environment, ensures regulatory convergence in the open and warehousing environments.

It improves application performance based on data provided by the business system as well as combining fine control of RFID technology. The application is important for changing the operating approach, enhancing management level, improving basis of operating efficiency, improving asset quality, extending equipment life, and optimizing network asset costs.

\section{Proposed RFID Application Model Based on Cyber-Physical Systems Architecture}

Real Time Location Systems are a kind of positioning method based on radio signal, using active RFID technology. Other indoor positioning technologies include passive RFID, 


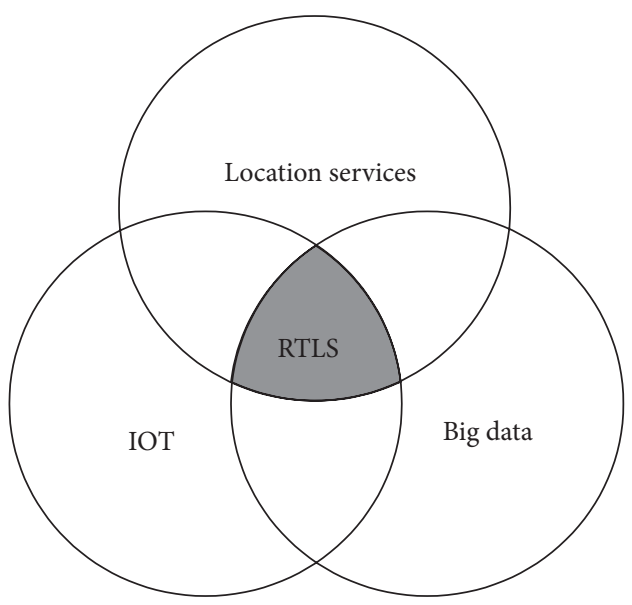

FIGURE 1: RFID-based real-time location system.

Wi-Fi and UWB. The pioneering research of social network [1], complex network [2-5], big data [6-11], and cyberphysical systems (CPS) [12-16] is emerging, which change the understanding of large-scale systems as in Figure 1 shows. CPS is the integration of computing and physical systems, which is the next generation of intelligent system to integrate computing, communication, and control.

In practice, techniques combination and application innovations can realize the effective management of assets physical state. Technique combinations include RTLS, RFID, and sensor technology. Application innovations guide the scheme for application through the research on RFID application model based on CPS architecture including acquisition, transmission, analysis, and decision making.

2.1. Proposed System Structure Model. The large enterprises' various RFID applications demand is the main factor; thus the RFID Application Model is proposed as Figure 2 shows. The model is divided into perception, transmission, calculation, control, and the supporting unit to form a complete closed-loop system, wherein the calculating unit includes a variety of real-time processing engine. This model meets the design requirements of the corporate assets applications and open environment applications.

Considering the huge amounts of data, the system needs to be configured flexible computing unit, capable of handling multidimensional data associated with the local real-time processing, timing, and behavior change information, including the location and spatial information and flow data, as well as the internal state and personnel state behavior. In short, the design model needs to meet the design requirements of the system in the context of the development of the Internet of Things and big data.

Considering the location status, time and other factors, the function $C(P, H, x, y, t)$ of the application model is established, as shown in the formula (1). Through the associated analysis of the position, status, and other data within the predetermined time, the preset rules for real-time behavioral analysis engine are obtained as the initial conditions of the model function. Subsequently, using the function and initial conditions, the completeness of the information recorded is formed:

$$
\begin{aligned}
& C(P, H, x, y, t) \\
& \quad=P(x, y, t)+\Delta P(t)+H(x, y, t)+\Delta S(t) .
\end{aligned}
$$

In the function, $P(t)$ represents the device location and $H(t)$ represents staff position; $\Delta P(t)$ represents a relative position; $\Delta S(t)$ represents a change of state; $(x, y, t)$ represents the two-dimensional position coordinates and time.

2.2. Analysis for the Comparative Model. Based on IoT application model, Big Data technology helps us for deep relationship. The important difference compared to the previous design is that the results and forecast information is quickly obtained through the phenomena associated with the data analysis, and there is no need to spend too much time to get the answer for the problem causal association. Traditional demand models tend to seek causal association, looking for trends, cycles, and other factors in order to create the function. For example, the user application requirements are described through the method of a random time series, as shown in the following formula

$$
Y(t)=f(t)+p(t)+X(t) .
$$

In the formula (2), $t$ represents time and $f(t), p(t)$ are nonrandom items. $f(t)$, the trend term, reflects the trend of the model $Y(t)$ which is changed by linear or exponential function; $p(t)$, the periodic term, reflects the cyclical change of $Y(t)$, such as year, month, day, or hour periodically; $X(t)$ is a random term, which reflects the impact of various random factors on $Y(t) . X(t)$ can be assumed to be a normal stationary random process. The $f(t)$ and $p(t)$ is generally not constant, and therefore $Y(t)$ is a nonstationary random process. Based on the above principles, nonrandom items $f(t)$ and $p(t)$ can be obtained through the acquisition system, and the statistics can be obtained through mathematical methods. Based on the type of user behavior, random item $X(t)$ can be accurately adjusted and standardized, and therefore $Y(t)$ has the higher accuracy, thereby forming a quasi-demand real-time model.

In the formula (1), although each item is random one, through the analysis of random mass data, certain patterns of behavior can be extracted as the analysis basis of the realtime behavior. Massive data analysis does not mean the way of the traditional mathematical statistics for obtaining samples, which emphasizes that the complete data is required as the basis for application, and it does not mean that the analysis of $X(t)$ from the formula (2) copied to $f(t)$ and $p(t)$ can get the result of the formula (1). There is a fundamental change, and the data is a whole complete collection.

The design procedures of the real-time behavior analysis engine come from the early accumulation and effective analysis of data. This model requires a certain modeling time T0 before the quasi-real-time system processing and will finally be able to get the key prediction model. The worth considering factors are as follows: (1) pay attention to the relative relationship, combined with the characteristics of RFID technology; (2) collect contact data as the basis for modeling future behavior. 


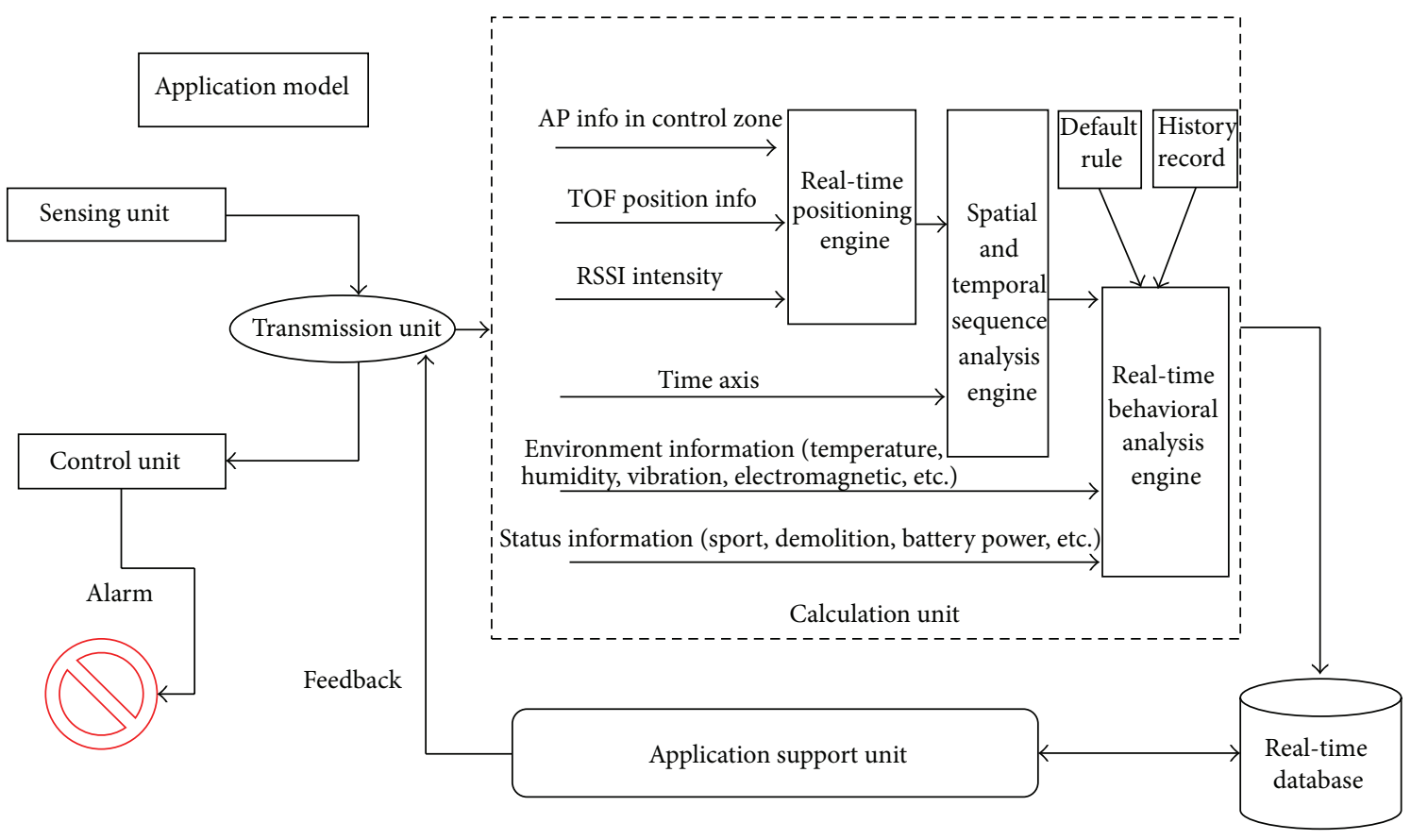

FiguRE 2: System structure model.

2.3. Consistency Analysis of Large-Scale Enterprise RFID Applications. In addition to the reliability and effectiveness of the system, consistency is a major consideration. The reference architecture depicts the overall application, wherein each unit division also has contact with each other. Each unit can be implemented to make a product, or can be used as a collection of several products, or part of a product. Despite the fact that the manufactured products have reached some kind of consistency request or meet the reliability under certain scenarios, the actual system need pay attention at the system level due to the change of scene, the integration of a variety of technologies, and products. Using the unified model and a simple combination of technologies can easily ensure application consistency, reliability, and validity.

\section{RFID Application Analysis and Solutions}

3.1. Problems and Demands for Data Asset Management. The data assets precise management requirements include: coordination and management of personnel and equipment based on traditional RFID asset management applications, management for differentiated IT assets and dynamic environment equipment, access to the status and location identification, solving communication interference in small closed environment, the use of a simple and reliable means of communication to reduce the procurement, and low costs of installation and maintenance.

3.2. Key Design for Data Asset Management. Integration of real-time positioning technology, multifrequency communication technology, sensor technology, assets, and personnel indoor mobile positioning is proposed to use. The dual-band communication technology realizes the static positioning

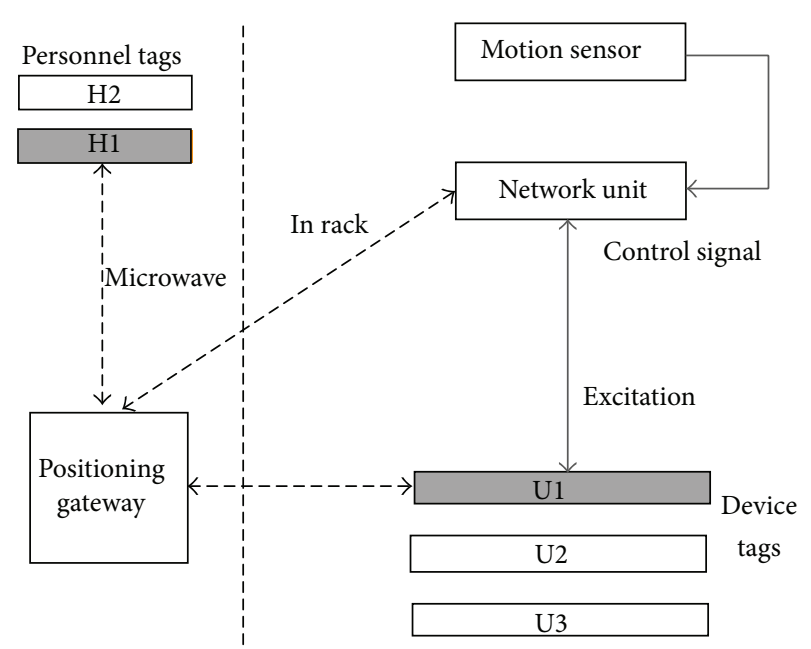

FIGURE 3: Design on RFID sensor application in real time positioning.

and situational awareness of local environmental assets. Integration of sensors, real-time location technology realizes equipment, personnel, mobile monitoring, and indoor navigation as shown in Figure 3.

Important components include the following: (1) motion sensor unit: integrated displacement and motion sensor, to complete the door opening and closing judgment and to activate the low-frequency two-way communication of low-frequency transceiver unit with cabinet asset locator tab; (2) low-frequency transceiver unit: completing cabinet positioning communications and moving the collected information to the network unit and also can be part of network unit; (3) network unit: integrated microwave band 
RFID communication module, with low frequency control module, sound and light alarm module; providing dynamic positioning with $2.4 \mathrm{G}$ microwave signal transmission, controlling the low-frequency transceiver unit with bidirectional communication; making the alarm and real-time feedback to remind the manager of job exceptions occurred in asset management; (4) positioning tag: integrated the active RFID positioning module with the low-frequency communication module to complete the $2.4 \mathrm{GHz}$ microwave band RFID positioning and low frequency positioning in rack; integrated motion/temperature/humidity sensor, sensing the movement status of the device and the cabinet temperature and humidity environment; (5) personnel tags: integrated active RFID positioning module to complete the $2.4 \mathrm{GHz}$ microwave band RFID location; integrated motion sensors for perception of staff state; (6) positioning gateway: realizing communication with network unit and the RFID tag, being as intelligent front-end unit of the asset monitoring application, running embedded systems, achieving the transmission of location data to background systems and databases.

The switch displacement sensor and low-frequency transceiver unit are installed inside the cabinet. Opening and closing movements trigger the interaction between positioning of labels and the cabinet gateway outside. According to the changes of the environment and equipment state, it is divided into three states: the cabinet opening with the device in stationary state, the device in moving state, and the cabinet closed with the device in stationary state. When the cabinet is turned on and the device is stationary, the cabinet network unit starts communication with the positioning gateway, just updating records and collecting gateway control commands. Under the motion state of the device, the RFID positioning function activated by the tag motion sensor is realizing positioning management in meter level accuracy, using a rack network unit as an auxiliary landmark. As cabinets closed with the device in still, the lower frequency signal transmission is fulfilled by low-frequency transceiver unit and tags then to be unified for communication by network unit and positioning gateway. Networking unit and positioning labels with temperature and humidity sensors constitute environment perception network inside cabinets, and the different cabinet network units form the upper senior-aware network with each other.

In the function (1), the device location $P(t)$ with relative position $\Delta P(t)$ and personal position $H(t)$ is realized by Tag and Low-frequency transceiver unit; the state information of the mobile sensing unit acts as $\Delta S(t)$. Positioning gateway has a computing unit functions.

The position information and the environment perception are mainly realized by hardware, that is, positioning gateway, sensor tag, and low-frequency transceiver unit. While the perception of the state, such as temperature and humidity, is achieved by the integration of special sensors. The design uses active RFID positioning technology and the integration of sensors of various types to achieve equipment location and status perception, which is cost-effective application of the cabinets unit and positioning sensor tag.

The simple design of the sensor could judge the state of the equipment and staff behavior. The data analysis and

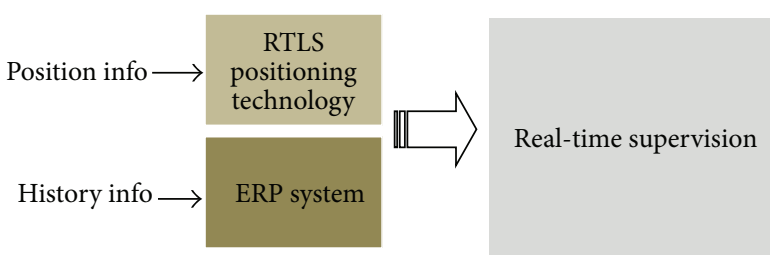

FIGURE 4: Vehicles battery supervision using RTLS and ERP.

threshold setting from the use of location data meet the design requirements of the application model well.

\subsection{Problems and Demands for Open Environment Battery} Asset Management. The battery storage management can refer to similar data asset management scheme described above. The main difference in warehouse management is the battery maintenance of large amounts of exchange data, roughly in the tens to hundreds of megabytes. The data transmission is the major demand in storage place densely stacked. Only a small amount of data is returned to the operator in the course, on account of the use of CAN and trip computer in conjunction with the mobile communication network, with the amount of data far less than the static data exchange and collection frequency being limited.

\subsection{Critical Designs for Open Environment Batteries Asset} Management Scheme. Battery storage management has different demands from data asset management, focusing on data delivery to the exclusion of networking, positioning, and collaborative technology. It uses active RFID to complete the Wi-Fi channel open and closed to achieve data transmission. Figure 4 shows vehicles battery supervision need the combination of RTLS and ERP.

RFID is not only identification achieved but also an excitation switch to realize the specified battery WIFI channel established. In the actual process, tens to hundreds of battery management communication can be accomplished only with several IP addresses. Using RFID as the management of the address and the communication channel solves the single battery status data exchange problem, which is a small proportion of the cost for valuable equipment.

In the operating environment, the battery status information collected by the battery management system will be transmitted to the remote by CAN, ECU and on-board computer shown as Figure 5. Environmental status of the battery in the car can be achieved using low-frequency communication. Associated together with cars, $P(t)$ is realized by GPS or GSM, and $\Delta S(t)$ is obtained by RFID sensor.

\subsection{Summaries for Battery Assets Management Practice}

(1) WIFI excitations realize detections of the battery in the shelf.

(2) DTU monitoring with GPRS dealing with the battery data transmission.

(3) Battery status collected by BMS is transmitted by the CAN bus to the vehicle terminal. 


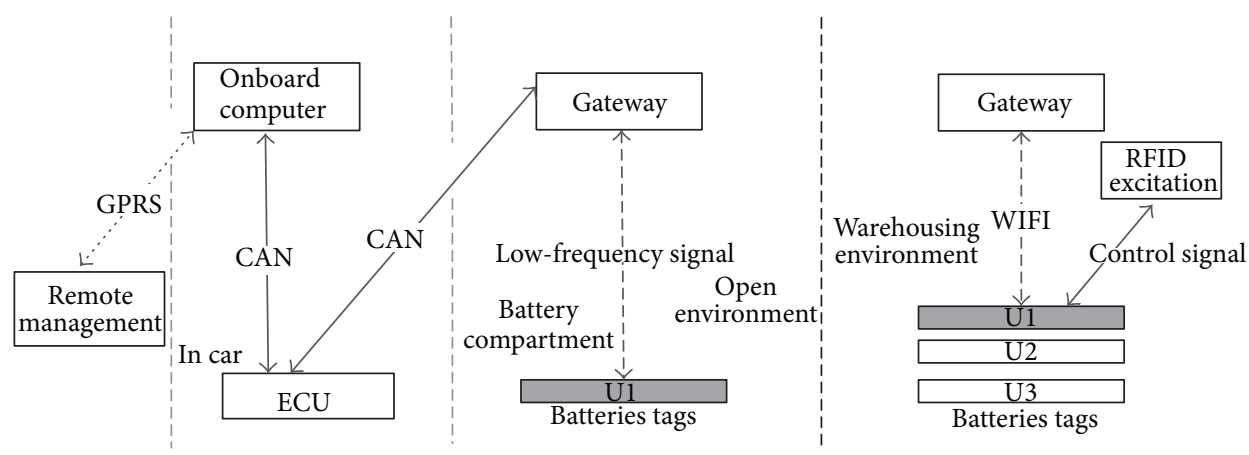

FIGURE 5: Design on RFID sensor application for battery assets.

\section{Analysis for Application Practice}

At present, the existing typical implementations include (1) passive UHF RFID application, using cabinet near field antenna design and installation location information, realizing equipment inventory and real-time location, is the case with patented product for data center; (2) active RFID and rack infrared integrated application: the solution can be viewed as the integrated application of active RFID and sensor technology. Real-time location-based applications of the microenvironment of the data center do not depend on a single positioning technology advanced but select and define the product by the actual needs.

(1) Passive RFID Design Defects. According to the comparison between design and model, the relative positional $P(t)$ is primarily obtained on the discrete-time $T 1, T 2$, and $T 3$, and so forth. Since adjacent time difference is not the same, along with the lack of temporal association and relationship of state and behavior, the design does not fully comply with the model. In addition, passive RFID location-based applications need to deploy a large number of antenna and reader, and the number of antenna elements cannot be changed, although it is possible to reduce the reader number by port splitters.

(2) Active RFID Scheme Design Features. Based on the above, relative position $\Delta P(t)$ is additionally used with discretetime changed into real-time and position increases precisely. Despite the timing relationship, the lack of the status and behavioral variables do not fully comply with the model, but the application has been relatively close.

According to application data characteristics of the electric vehicles services platform, operation data can be divided into basic data, information file data, processing data, periodic data, documents data, and statistics query data. Currently, big data with real-time sensor data is the new application. The new practical experiment data would be collected and presented to justify the practice of these proposed models.

\section{Advantage of Applications from the Model}

The change in location, timing, and behavior is as the information input. The relative position and area position from active RFID form the basis of the space-time cube geospatial data. Staff position change improves the interactivity, using multidimensional and massive data modeling as the formation of real-time applications. The effectiveness and consistency is improved compared to traditional RFID systems. In practical applications, the selection of passive RFID, active RFID, and RFID with sensors tends to the third which has obvious application value due to the fact that the prior two are the actual product, and the last is applied to the actual project.

5.1. Changes for IT Assets Management Application. Realtime positioning sensors can take into account dynamic, static, and cabinet-level position identification. The integrated innovation in application mode based on behavioral analysis can be achieved. For example, classified usage rules of tags created, the function in each tag dynamic changes. Classification model is established based on the usage frequency of the tags, and taking advantage of middleware for the classification management function in Figure 6, the different units of tag can dynamic wake up and close. The specific design includes priority for critical equipment to use active tags; combined with sensor, increasing the equipment motion recognition; the combination of video and active tags to increase staff identification and behavior monitoring; realtime collaborative management of the operation of the device and persons. In addition, the policy is applied mainly for the classification of the cabinet-level identification. Just the cabinet level monitoring with simplified static positioning accuracy and mobile positioning can record formation of $C(P, H, x, y, t)$ to form an effective monitoring.

5.2. Benefit of Vehicle Battery Application. The importance of electric vehicle batteries is obvious, the potential value of which is the life cycle management, and it is extremely important for operator to obtain comprehensive data as commercial design using RFID hardware infrastructure at different stages. From the perspective of standard and industrial development, we still require the reliable design to meet electric vehicles battery needs of production, leasing and operations. However, for State Grid battery management, it is already an important attempt. 


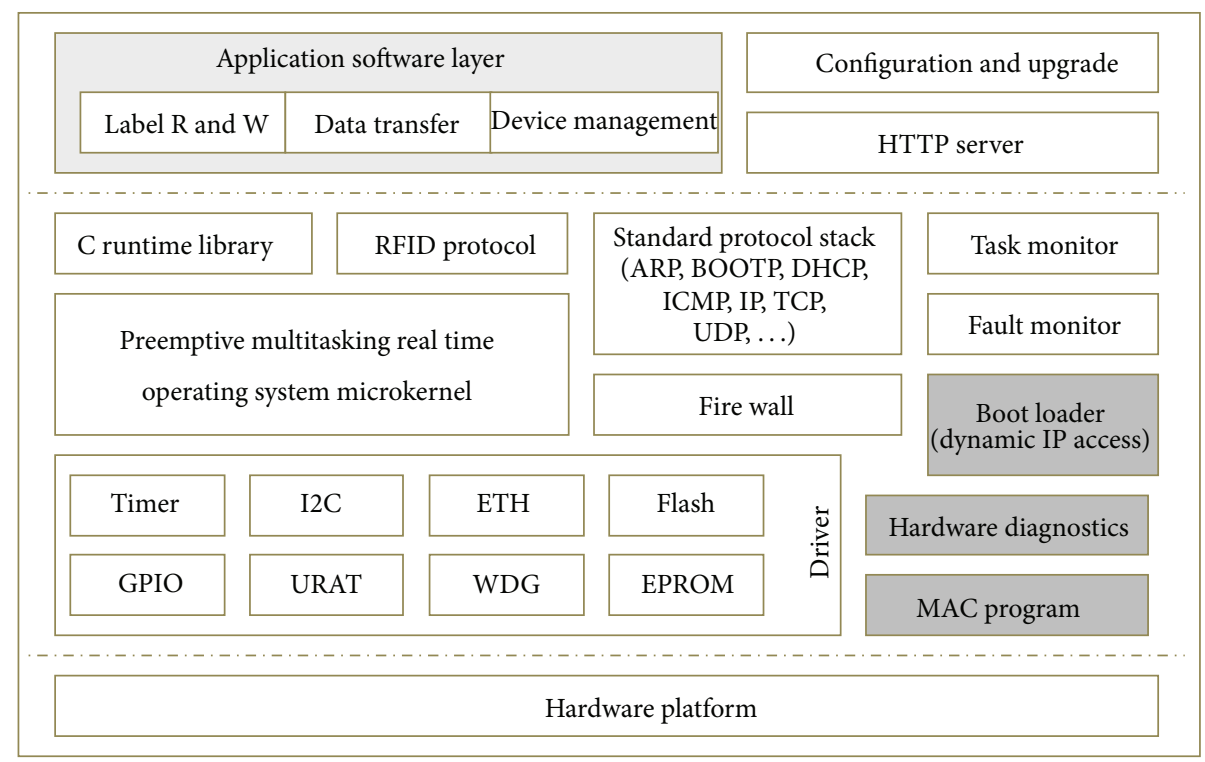

FIGURE 6: Front-end software infrastructure of RFID applications.

Electric vehicle asset management and IT assets both take advantage of RFID technology to enhance the reliability, security, and trace ability of asset. The difference between data center application and battery management is that the former is applied to closed environment and the latter is applied in complex open environment.

\section{Conclusions}

In the current social environment and development opportunities, taking advantage of RFID and other technologies, as well as the practical demand-driven, the new application ecological system is being formed; some of which are local for enterprises management and the others are open applications that are beneficial to social services, to promote RFID continuous development.

\section{Conflict of Interests}

The authors declare that there is no conflict of interests regarding the publication of this paper.

\section{References}

[1] S. Milgram, “The small world problem," Psychology Today, vol. 2, no. 1, pp. 60-67, 1967.

[2] R. Cohen and S. Havlin, "Scale-free networks are ultrasmall," Physical Review Letters, vol. 90, no. 5, Article ID 058701, 4 pages, 2003.

[3] R. Albert, H. Jeong, and A. Barabási, "Error and attack tolerance of complex networks," Nature, vol. 406, no. 6794, pp. 378-382, 2000.

[4] D. J. Watts and S. H. Strogatz, "Collective dynamics of "smallworld” networks," Nature, vol. 393, no. 6684, pp. 440-442, 1998.

[5] J. M. Kleinberg, "Navigation in a small world," Nature, vol. 406, no. 6798 , p. $845,2000$.
[6] A. J. G. Hey, The Fourth Paradigm: Data-Intensive Scientific Discovery, 2009.

[7] D. Howe, M. Costanzo, P. Fey et al., "Big data: the future of biocuration," Nature, vol. 455, no. 7209, pp. 47-50, 2008.

[8] J. Dean and S. Ghemawat, "MapReduce: simplified data processing on large clusters," Communications of the ACM, vol. 51, no. 1, pp. 107-113, 2008.

[9] C. Anderson, "The end of theory: the data deluge makes the scientific method obsolete," Wired, 2008.

[10] S. Ghemawat, H. Gobioff, and S. T. Leung, "The Google file system," ACM SIGOPS Operating Systems Review, vol. 37, no. 5, pp. 29-43, 2003.

[11] L. Guojie and C. Xueqi, "Research status and scientific thinking of big data," Strategy and Policy Decision Research, vol. 6, pp. 647-657, 2012.

[12] E. A. Lee, "Cyber physical systems: design challenges," in Proceedings of the 11th IEEE Symposium on Object-Oriented Real-Time Distributed Computing (ISORC '08), pp. 363-369, Orlando, Fla, USA, May 2008.

[13] Y. Tan, M. C. Vuran, and S. Goddard, "Spatio-temporal event model for cyber-physical systems," in Proceedings of the 29th IEEE International Conference on Distributed Computing Systems Workshops (ICDCSW'09), pp. 44-50, 2009.

[14] J. E. Kim and D. Mosse, "Generic framework for design, modeling and simulation of cyber physical systems," $A C M$ SIGBED Review, vol. 5, no. 1, pp. 1-2, 2008.

[15] R. R. Rajkumar, I. Lee, L. Sha, and J. Stankovic, "Cyber-physical systems: the next computing revolution," in Proceedings of the 47th Design Automation Conference (DAC '10), pp. 731-736, ACM, June 2010.

[16] H. Ahmadi, T. F. Abdelzaher, and I. Gupta, "Congestion control for spatio-temporal data in cyber-physical systems," in Proceedings of the 1st ACM/IEEE International Conference on CyberPhysical Systems (ICCPS '10), pp. 89-98, ACM, April 2010. 

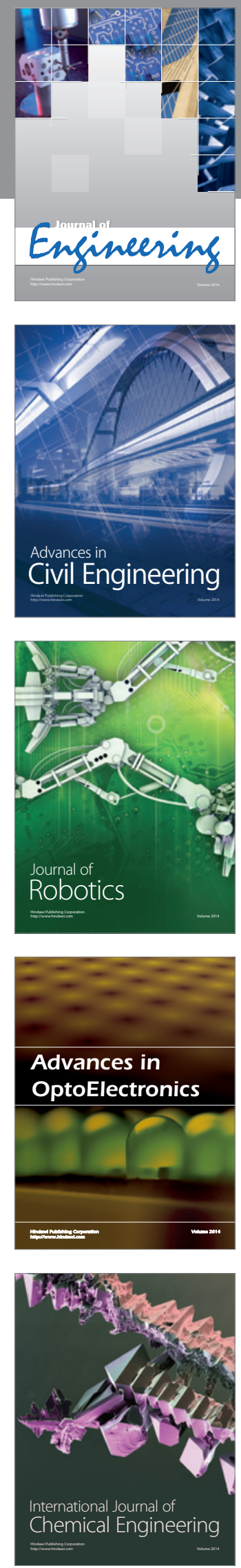

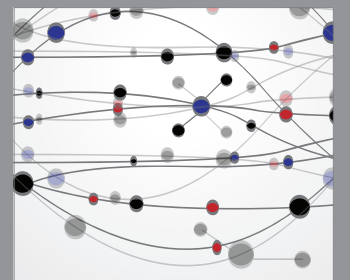

The Scientific World Journal
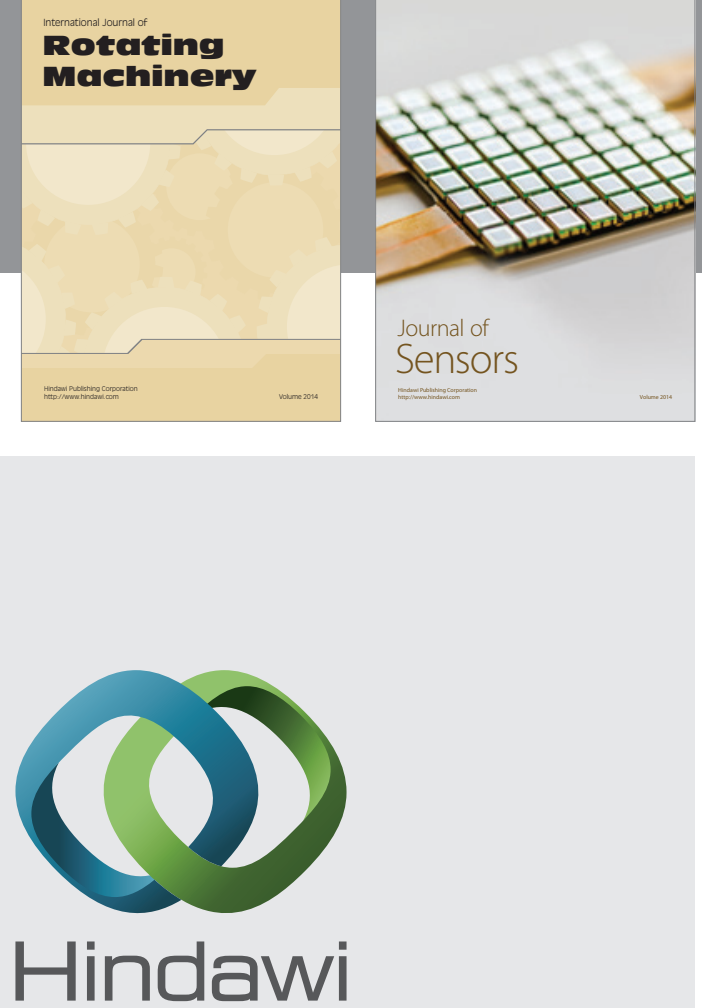

Submit your manuscripts at http://www.hindawi.com
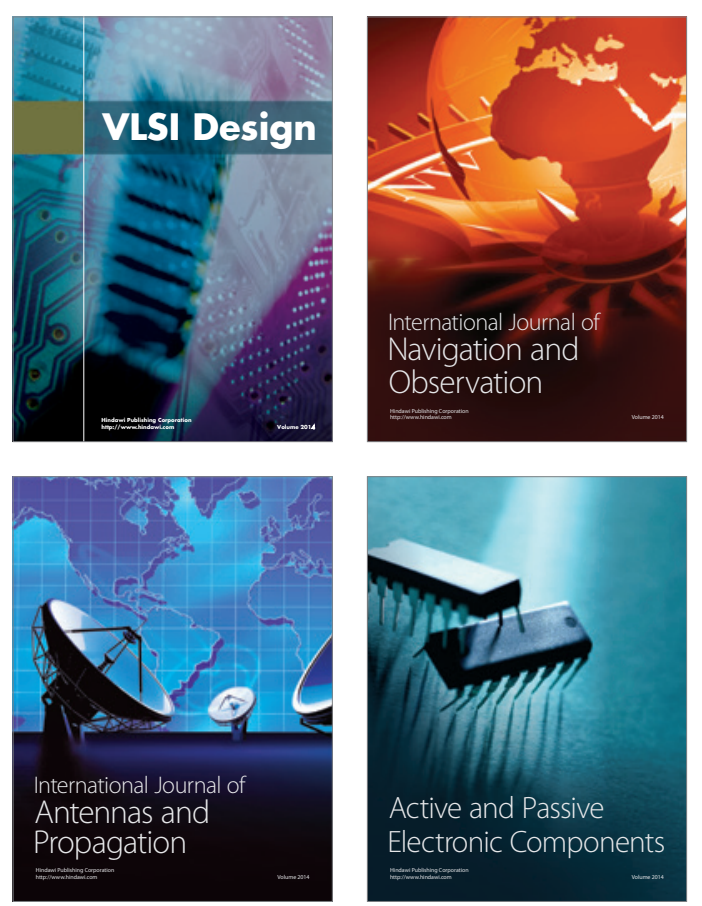
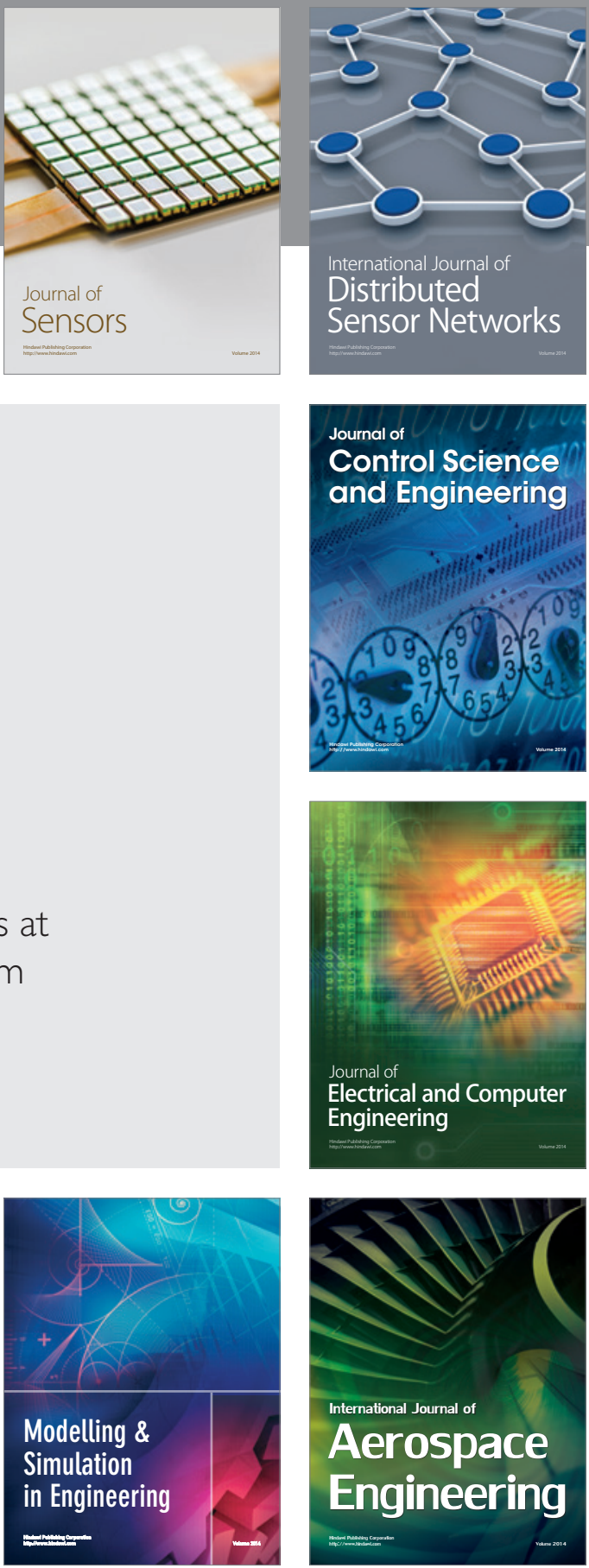

Journal of

Control Science

and Engineering
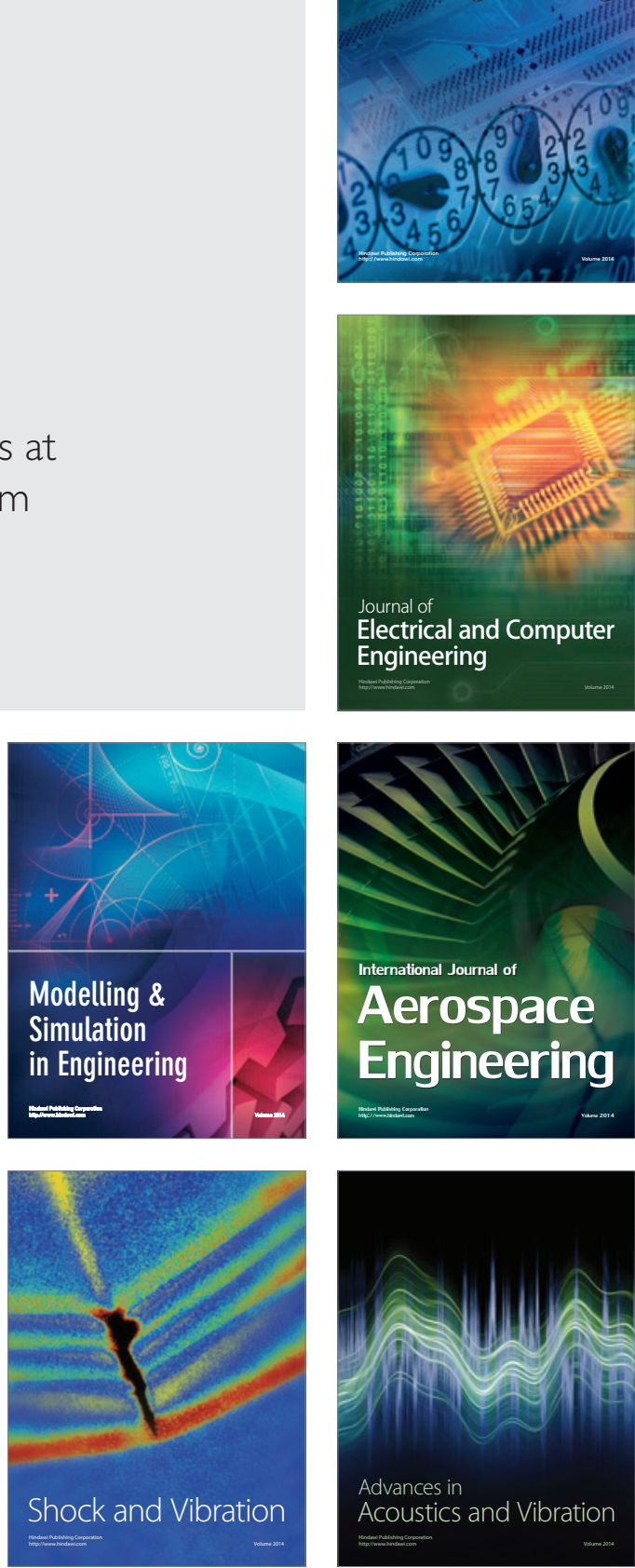